\section{Commentary: The educational hole is no bigger than the sum of its parts}

\author{
David L. Joyce, MD, MBA \\ That's all well and good in practice, but how does it \\ work in theory?
}

\author{
—Schmuel Weinberger
}

Residency training in the 21 st century requires the mastery of increasingly complex skill sets in the context of restrictions on the number of allowable work hours and years of experience logged in preparation for the board-certification exam. Among the innovative approaches that have been proposed to address this obvious gap, simulation of surgical procedures has emerged as a clear favorite of program directors and trainees alike. ${ }^{1}$ In their thoughtful approach to teaching what may be the most fundamental of cardiac surgical skills, Vo and colleagues ${ }^{2}$ demonstrate an approach to education that the members of our specialty would do well to emulate.

Among the immediate questions that arises upon considering a simulation model for median sternotomy involves the availability of viable substitutes-namely realworld experience. Presumably the expanding implementation of transcatheter and robotic therapies has not completely eliminated the educational opportunities for perfecting this skill on a live human. To understand why we need a sternotomy simulator, one must consider the 3 criteria which must be satisfied for a resident physician

\footnotetext{
From the Division of Cardiothoracic Surgery, Department of Surgery, Medical College of Wisconsin, HUB for Collaborative Medicine, Milwaukee, Wis.

Disclosures: The author reported no conflicts of interest.

The Journal policy requires editors and reviewers to disclose conflicts of interest and to decline handling or reviewing manuscripts for which they may have a conflict of interest. The editors and reviewers of this article have no conflicts of interest.

Received for publication Feb 8, 2020; revisions received Feb 8, 2020; accepted for publication Feb 20, 2020; available ahead of print April 22, 2020.

Address for reprints: David L. Joyce, MD, MBA, Division of Cardiothoracic Surgery, Department of Surgery, Medical College of Wisconsin, HUB for Collaborative Medicine, 8701 Watertown Plank Rd, Milwaukee, WI 53097 (E-mail: djoyce@ mcw.edu).

JTCVS Techniques 2020;2:119-20

2666-2507

Copyright (C) 2020 The Authors. Published by Elsevier Inc. on behalf of The American Association for Thoracic Surgery. This is an open access article under the CC BY-NCND license (http://creativecommons.org/licenses/by-nc-nd/4.0/).

https://doi.org/10.1016/j.xjtc.2020.02.036
}

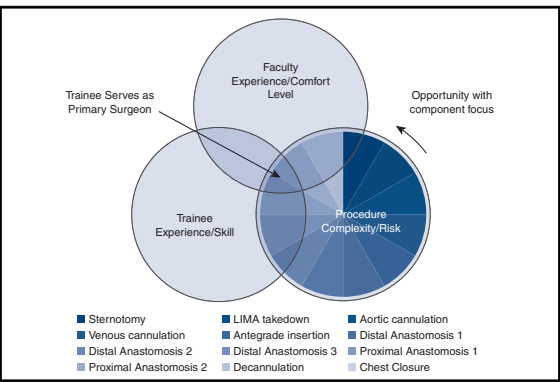

Breaking down surgical maneuvers into components improves resident experience and feedback.

\section{CENTRAL MESSAGE \\ Resident operative experience can be enhanced through a component-based teaching emphasis that maximizes oppor- tunities to exposure to even the most complex cases.}

to perform an operation as primary surgeon (Figure 1): The faculty member must have enough experience and confidence in performing the procedure that adding a trainee to the mix will not jeopardize the outcome of the patient, the trainee must have an appropriate level of experience and skill to perform the operation without an increased risk of complications, and the procedural complexity and risk must allow for the extra time that is often needed in the context of a teaching case. It is within this framework that the present study draws our attention to what may be perhaps among the most glaring weaknesses of the current paradigm. Resident physicians are required to $\log$ cases to qualify for the board examination. Perhaps our focus should instead be on components. ${ }^{3}$ An intern may be unqualified to place every suture on a Loeys-Dietz valve-sparing aortic root replacement but surely there would be a lower threshold for teaching this same trainee how to perform a sternotomy on the same patient? Imagine a component logging system that would inform the expectations of faculty members based on the number of sternotomies performed, aortas cannulated, and coronary button anastomoses performed by an individual resident.

Of course, even in an optimized teaching environment in which residents always maximize their operative experience by performing each component appropriate to their skill and experience level, there will still be a first time for every operative maneuver. It is for this reason that the 


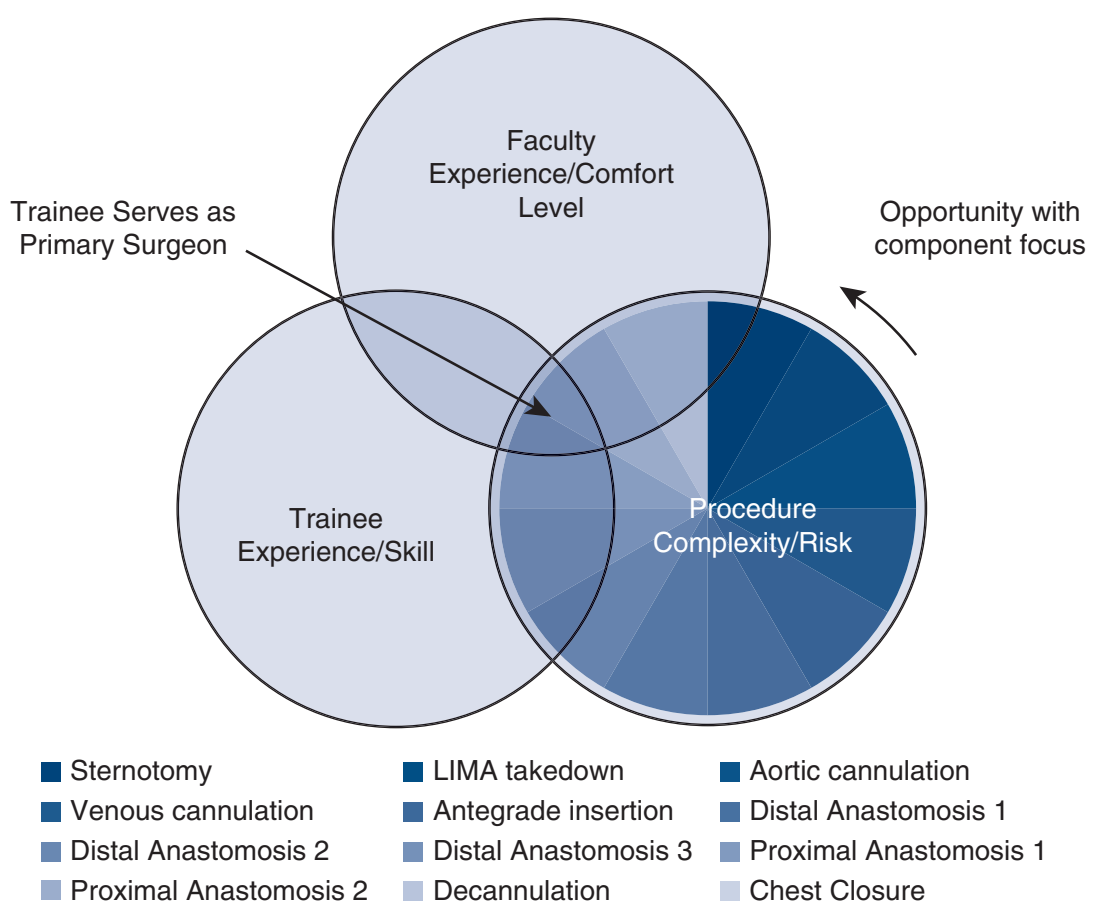

FIGURE 1. Breaking down surgical maneuvers into components improves resident experience and feedback.

protocol outlined in the current study provides valuable benchmarks for those inexperienced in handling a sternal saw. The checklist Vo and colleagues ${ }^{2}$ provide in their online-only material will become immediate mandatory reading for junior residents in my own operating room, and I suspect that codifying even this most basic of techniques will prove valuable to educators and trainees alike.

\section{References}

1. Connors RC, Doty JR, Bull DA, May HT, Fullerton DA, Robbins RC, et al. Effect of work-hour restriction on operative experience in cardiothoracic surgical residency training. J Thorac Cardiovasc Surg. 2009;137:710-3.

2. Vo TX, Juanda N, Ngu J, Gawad N, LaBelle K, Rubens FD, et al. Development of a median sternotomy simulation model for cardiac surgery training. J Thorac Cardiovasc Surg Tech. 2020;2:109-16.

3. Ericsson KA. Deliberate practice and acquisition of expert performance: a general overview. Acad Emerg Med. 2008;15:988-94. 